\title{
Manual auto instructivo "TCl" para mejorar la calidad de los trabajos de investigación monográfica de los estudiantes de segundo año de la UNJBG Tacna-2012
}

The auto instructive manual "TCl" for improves the quality of monographic investigation works of the students of from students of second year of the UNJBG Tacna - 2012

Nancy Apaza Chambilla ${ }^{1}$

\section{RESUMEN}

Objetivo: Plantear y validar el manual auto instructivo "TCl" como estrategia didáctica para mejorar la calidad de los trabajos de investigación monográfica de los estudiantes de segundo año de LELI de la Escuela Académica Profesional de Educación de la UNJBG de Tacna en el año académico 2012.

Método: La investigación es de tipo aplicada, de naturaleza cuasi experimental, interviene en la variable dependiente: calidad de los trabajos de investigación monográfica. Para lo cual, se consideró la información obtenida a través de la aplicación Manual auto instructivo "TCl", el cual fue aplicado al grupo experimental, constituido por 30 estudiantes de la Escuela Académica Profesional de Educación de la especialidad de Lengua y Literatura (LELI) de segundo año académico de la Universidad Nacional Jorge Basadre Grohmann (UNJBG) de Tacna.

Resultado: El manual auto instructivo "TCl" es una estrategia didáctica para mejorar la calidad de los trabajos de investigación monográfica de los estudiantes, tal como se aprecia en los resultados del grupo experimental (GE) de la presente investigación; con lo cual, además se demuestra la validez del manual.

Conclusión: el manual auto instructivo "TCl" repercute en forma significativa en la calidad de los trabajos de investigación monográfica de los estudiantes de segundo año de la Escuela Académica Profesional de Educación de LELI de la UNJBG de Tacna en el año académico 2012.

\section{PALABRAS CLAVE}

Calidad, investigación monográfica, manual auto instructivo "TCl", internet.

\begin{abstract}
Objetive: Propose and validate self-instructional manual "TCl" as a teaching strategy to improve the quality of the work of monographic research sophomores LELI of Vocational Academic Education School UNJBG of Tacna in the 2012 academic year.

Method: The investigation is of applied type of quasi-experimental nature intervenes in the dependent variable: quality of works monographic research. For this purpose, the information obtained through the manual application auto instructive "TCl" which was applied to the experimental group, consisting of 30 students from the Professional Academic School of Education specializing in Language and Literature (LELI) was considered second academic year of Jorge Basadre Grohmann National University (UNJBG) of Tacna.

Result: Self-instructional manual "TCl" is a teaching strategy to improve the quality of the work of monographic research students, as seen in the results of the experimental group (EG) of the present investigation; thereby also the validity of the manual shows.

Conclusion: self-instructional manual "TCl" significantly affects the quality of work of monographic research sophomores Academic School of Vocational Education UNJBG LELI of Tacna in the 2012 academic year.
\end{abstract}

\section{KEYWORDS}

Quality, monographic investigation, auto instructive manual "TCl", internet. 


\section{Introducción}

Siglo XXI es la era de internet, pues se ha convertido en el medio idóneo para impartir una enseñanza de calidad y de progreso no solo para la empresas con fines económicos, sino para las instituciones educativas. Sin duda alguna, uno de los avances más importantes de los últimos tiempos ha sido "Internet", esta ha surtido su efecto desde la ciencia, la política, el arte, hasta en la educación y posibilitando según Wyatt, A. (1995), que miles de personas con solo tener una computadora y una conectividad a internet, puedan acceder a todo un mundo de posibilidades al alcance de sus manos. Es en este sentido, que Internet es una herramienta importante en la educación, cambiando la manera de enseñanza - aprendizaje, donde el individuo interactúa con sus pares, y es, él mismo, el protagonista de su aprendizaje, dejando de lado el papel de sujeto pasivo y tomando un rol activo en su educación.

Internet permitió que el conocimiento se descentralizara del aula y estuviera en el ciberespacio, abriendo las ofertas educativas a las personas sin límites de tiempo y espacio, y lo más importante, el usuario no debe desplazarse, ni desarraigarse de su lugar de origen para poder acceder al conocimiento. Internet, hoy se emplea fundamentalmente como una nueva biblioteca.

Según Vara, A. (2010), los estudiantes utilizan Internet como fuente primaria, donde aplican sus criterios para la elaboración del trabajo de investigación monográfica. Asimismo, Vara, A. (2010) precisa "...se aprecia que no seleccionan, procesan y sistematizan la información adecuadamente...". En ocasiones, simplemente copian y pegan la información, por lo tanto, dichos trabajos resultan ser incoherentes y de deficiente calidad.

El manual auto instructivo denominado Trabajando con Internet (TCl) ofrece una serie de estrategias didácticas para mejorar y subsanar las deficiencias de los trabajos de investigación monográfica.

Siendo la pregunta de la investigación ¿En qué medida la aplicación del manual auto instructivo Trabajando con Internet (TCl) permitirá mejorar la calidad de los trabajos de investigación monográfica de los estudiantes de segundo año de Lengua y Literatura (LELI) de la Universidad Nacional Jorge Basadre Grohmann (UNJBG) de Tacna en el año académico 2012? Y se formula la siguiente hipótesis: La aplicación del manual auto instructivo "TCl" mejora significativamente la calidad de los trabajos de investigación monográfica de los estudiantes de segundo año de LELI de la Escuela Académica Profesional de Educación de la UNJBG de Tacna en el año académico 2012.

El trabajo de investigación presenta dos grupos, grupo control y grupo experimental. El primero corresponde a los estudiantes de segundo año de Idioma Extranjero, Traductor e Intérprete (IETI); mientras, que el segundo corresponde a los estudiantes de segundo año de Lengua y Literatura (LELI) de la Escuela Académica Profesional de Educación de la Universidad Nacional Jorge Basadre Grohmann (UNJBG). 
La investigación está estructurada en seis capítulos. En el capítulo Ise presenta la formulación del problema, justificación y objetivos de la investigación. En el capítulo II se aborda el tema del Manual Auto instructivo "TCl". En el capítulo III se desarrolla el tema del trabajo de investigación monográfica. En el capítulo IV se describe el marco metodológico empleado en la investigación. En el capítulo $\mathrm{V}$ se presentan los resultados de la investigación, las pruebas estadísticas y la comprobación de las hipótesis. Finalmente, en el capítulo VI se presentan las conclusiones $y$ sugerencias de la investigación.

La investigación titulada "Manual auto instructivo trabajando con internet (TCl) para mejorar la calidad de los trabajos de investigación monográfica de los estudiantes de segundo año de la especialidad de lengua y literatura (LELI) de la Escuela Profesional de Educación de la UNJBG, ciudad de Tacna, año 2012" constituye un aporte para el sector educativo, sobre todo, para garantizar y optimizar la elaboración de los trabajos de investigación monográfica de los estudiantes.

\section{Materiales y métodos}

El tipo de investigación es aplicada, porque tiene por objeto intervenir en la variable dependiente y lograr la modificación de su comportamiento.

Se aplicará el manual auto instructivo "TCl" para mejorar la calidad de trabajos de investigación monográfica. El diseño de investigación es cuasi experimental, donde GC
(Grupo de Control), GE (Grupo experimental) y $\mathrm{X}$ (Tratamiento Experimental). El ámbito de estudio es la Universidad Nacional Jorge Basadre Grohmann. El grupo de investigación está conformado por estudiantes de segundo año, de la especialidad de Lengua y Literatura (LELI) e Idioma Extranjero, Traductor e intérprete (IETI) del año académico 2012.

Las unidades de estudio son los estudiantes de la Universidad Nacional Jorge Basadre Grohmann (UNJBG) del año académico 2012, constituido por grupo control y grupo experimental. La población está constituida por los estudiantes que conforman las cinco escuelas profesionales: Lengua $y$ Literatura (LELI), Ciencias Sociales y Promoción Sociocultural (SPRO), Idioma Extranjero, Traductor e intérprete (IETI), Ciencias de la Naturaleza, Ecología y Ambiente (CNEA), Matemática y Computación (MACO). La muestra está constituida por los estudiantes de segundo año de Lengua y Literatura (LELI- 30 estudiantes) y estudiantes de Idioma Extranjero, Traductor e intérprete (IETI26 estudiantes). Se trabajó con grupos intactos.

Los datos se recopilaron de las fuentes primarias, es decir, de los trabajos de investigación monográfica de los estudiantes que integran el grupo control y grupo experimental. Para la recolección de los datos se utilizó la técnica documental y juicio de expertos. Se aplicó el pre test (evaluación de entrada) y post test (evaluación de salida). 
Con el primero, se precisa la categoría a la que corresponden los trabajos de investigación monográfica, tanto del grupo control como del grupo experimental. El segundo, permite comparar los resultados de la aplicación del "Manual Auto instructivo TCl" a fin de determinar los resultados. Se aplicó la ficha de análisis documental para la técnica documental con el fin de determinar la calidad de los trabajos de investigación monográfica, antes y después de la experiencia. Asimismo, se utilizó como instrumento la Ficha de validación de Juicio de expertos para evaluar la calidad y el impacto del manual auto instructivo "TCl". Los resultados se presentan mediante tablas y gráficos estadísticos. El análisis se basa en proporciones y razones; y para la interpretación de los resultados, así como para comprobación de la hipótesis, se trabaja con procedimientos lógicos.

\section{Resultados}

Diseño de presentación de los resultados

La información procesada se presenta según el siguiente orden:

- Información sobre la situación de la calidad de los trabajos de investigación monográfica antes de la aplicación del manual auto instructivo "TCl".

- Información sobre la situación de la calidad de los trabajos de investigación monográfica después de la aplicación del manual auto instructivo "TCl".

- Comprobación de las hipótesis.

\section{Presentación y análisis de los resultados}

La información de la calidad de los trabajos de investigación monográfica se presenta en base a dos dimensiones: forma y fondo.

Información sobre la situación de la calidad de los trabajos de investigación monográfica antes de la aplicación del manual Auto instructivo "TCl"

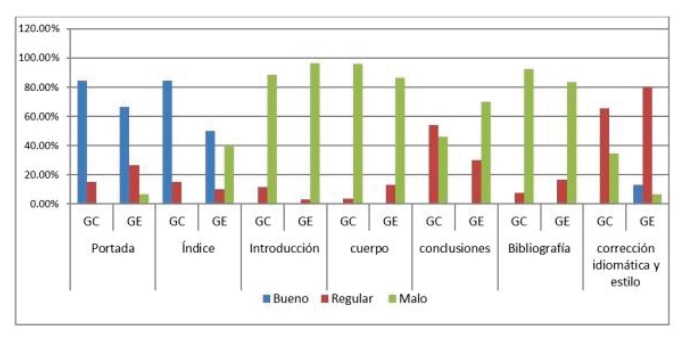

Figura 1.

Comparativo porcentual de la evaluación de los indicadores del trabajo de investigación monográfica del grupo control y grupo experimental (Evaluación de Entrada)

De acuerdo a la Figura 1, relacionada a la evaluación de entrada de los indicadores del trabajo de investigación monográfica del grupo de control y grupo experimental; se distingue que el indicador portada (GC=84.62\% y GE= $66.67 \%)$ e índice (GC $=84.62 \%$ y $\mathrm{GE}=50.00 \%$ ) se ubican en la categoría bueno; mientras que los indicadores introducción ( $\mathrm{GC}=88.46 \%$ y $\mathrm{GE}=$ $96.67 \%$ ), cuerpo (GC=96.15\% y $\mathrm{GE}=$ $86.67 \%$ ) y referencias bibliográficas $(\mathrm{GC}=92.31 \%$ y $\mathrm{GE}=83.33 \%)$ se sitúan en la categoría malo.

Los datos presentados, tanto en el grupo control y grupo experimental, muestran la presencia de dificultades en la introducción, cuerpo y referencias bibliográficas; y menores limitaciones en relación a la portada e índice. Asimismo, en conjunto, el grupo de 
control y grupo experimental son relativamente similares, aunque en algunos indicadores el grupo control supera al grupo experimental.

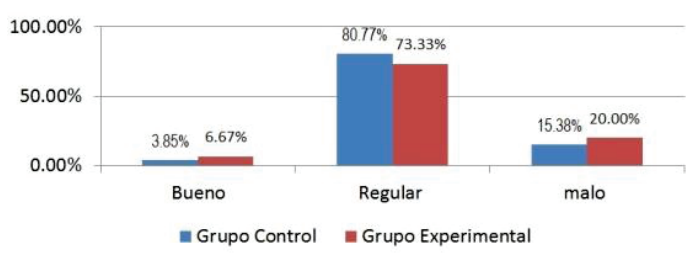

Figura 2.

Evaluación de entrada de la calidad de los trabajos de investigación monográfica del grupo control y grupo experimental, Tacna -2012

Según la Figura 2, relacionada a la evaluación de entrada del trabajo de investigación monográfica presentado por los estudiantes del grupo control y grupo experimental; se observa que el $3,85 \%$ del grupo control y $6,67 \%$ del grupo experimental se ubican en la categoría bueno, mientras que el $\quad 80,77 \%$ y $73,33 \%$ respectivamente se sitúan en la categoría regular. Asimismo, el $15,38 \%$ y $20,00 \%$ respectivamente se encuentran en la categoría malo.

La información presentada sugiere la presencia de mayores limitaciones en la introducción, el cuerpo, las conclusiones y referencias bibliográficas; y menores dificultades en la portada de la.

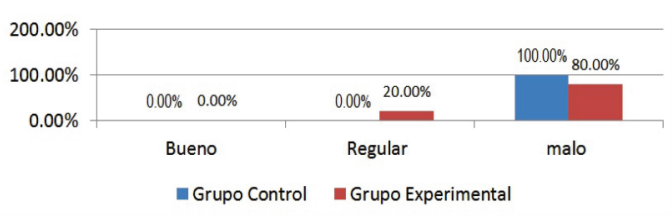

Figura 3.

Evaluación de entrada del fondo de trabajo de investigación monográfica del grupo control y grupo experimental, Tacna -2012
Según la Figura 3, concerniente a la evaluación de entrada del fondo del trabajo de investigación monográfica presentado por los estudiantes del grupo control y grupo experimental; se observa que el $0,00 \%$ del grupo control y $20,00 \%$ del grupo experimental se ubican en la categoría regular. Mientras que el $100,00 \%$ y $80,00 \%$, respectivamente, se sitúan en la categoría malo.

La información presentada indica la presencia de mayores limitaciones del grupo control en relación al fondo.

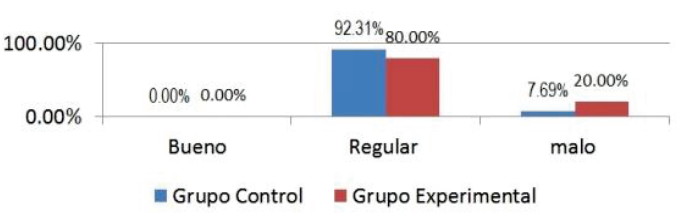

Figura 4.

Evaluación de entrada de la forma del trabajo de investigación monográfica del grupo control y grupo experimental, Tacna -2012

De acuerdo a la Figura 4, relacionada a la evaluación de entrada de la forma del trabajo de investigación monográfica presentado por los estudiantes del grupo control y grupo experimental; se precisa que el $92,31 \%$ del grupo control y el $80,00 \%$ del grupo experimental se ubican en la categoría regular; mientras que, el $7.69 \%$ y $20,00 \%$ respectivamente se sitúan en la categoría malo.

La información presentada muestra la presencia de mayores limitaciones del grupo experimental en relación a la forma.

Información sobre la situación de la calidad de los trabajos de investigación monográfica después de la aplicación del manual Autoinstructivo "TCl". 


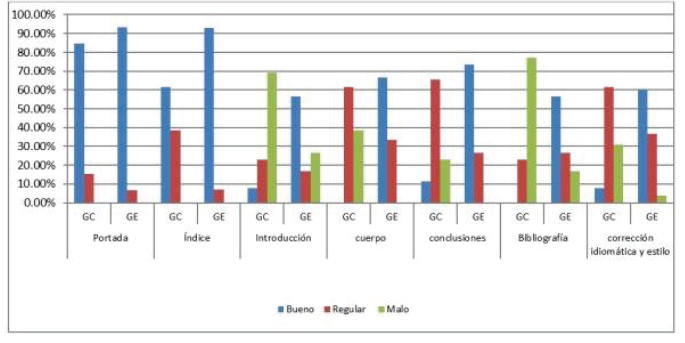

Figura 5.

Comparativo porcentual de la evaluación de salida de los indicadores del trabajo de investigación monográfica del grupo control y grupo experimental (evaluación de salida)

De acuerdo a la Figura 5, relacionada a la evaluación de salida de los indicadores del trabajo de investigación monográfica del grupo de control y grupo experimental; se aprecia que después de la experiencia los indicadores portada $(\mathrm{GC}=84.62 \%$ y $\mathrm{GE}=93.33 \%)$, indice $(\mathrm{GC}=61.54 \% \mathrm{y}$ $\mathrm{GE}=93.10 \%)$, cuerpo (GC $=0.00 \%$ y $\mathrm{GE}=66.67 \%)$ y conclusiones (GC=11.54\% y $\mathrm{GE}=73.33 \%$ ) se ubican en la categoría bueno. Por tanto, los indicadores citados han mejorado significativamente en el grupo experimental, mientras que el grupo de control mejora levemente en comparación de la evaluación de entrada (ver tabla $N^{\circ} 08$ ). Asimismo, los demás indicadores han elevado su nivel de calidad.

Los datos presentados del grupo experimental, en su conjunto, han mejorado significativamente en relación al grupo de control.

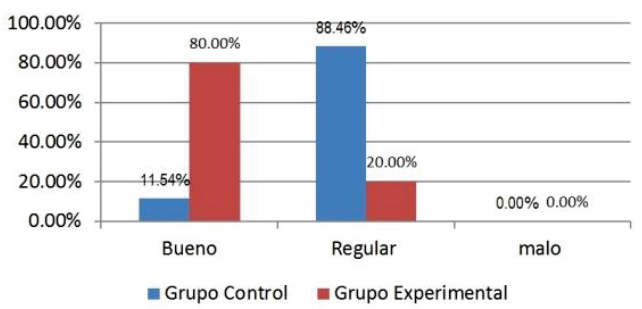

Figura 6.

Evaluación de salida del trabajo de investigación monográfica del grupo control y grupo experimental, Tacna - 2012.
Según la Figura 6, relacionada a la evaluación de salida del trabajo de investigación monográfica presentado por los estudiantes del grupo control y grupo experimental; se observa que el $11,54 \%$ del grupo control y $80,00 \%$ del grupo experimental se ubican en la categoría bueno; mientras que el $88,46 \%$ y $20,00 \%$ respectivamente se sitúan en la categoría regular. Asimismo, ningún trabajo se encuentra en la categoría malo.

La información presentada sugiere la presencia de mayores limitaciones en la introducción, tanto en el grupo control como en el grupo experimental. Además, los trabajos del grupo control presentan dificultades en la referencia bibliográfica. Finalmente, se aprecian menores dificultades en la portada de la investigación.

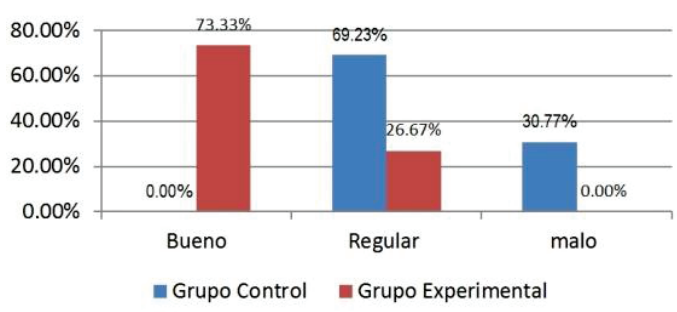

Figura 7.

Evaluación de salida del fondo del trabajo de investigación monográfica del grupo control y grupo experimental, Tacna - 2012.

Según la Figura 7 , concerniente a la evaluación de salida del fondo del trabajo de investigación monográfica presentado por los estudiantes del grupo control y grupo experimental; se observa que el $0,00 \%$ del grupo control y $73,33 \%$ del grupo experimental se ubican en la categoría bueno; mientras que el $96,23 \%$ y $26,67 \%$ respectivamente se sitúan en la categoría regular; y el $30,77 \%$ y $0,00 \%$ 
respectivamente se ubican en la categoría malo.

La información presentada manifiesta la presencia de mayores limitaciones del grupo control en relación al fondo.

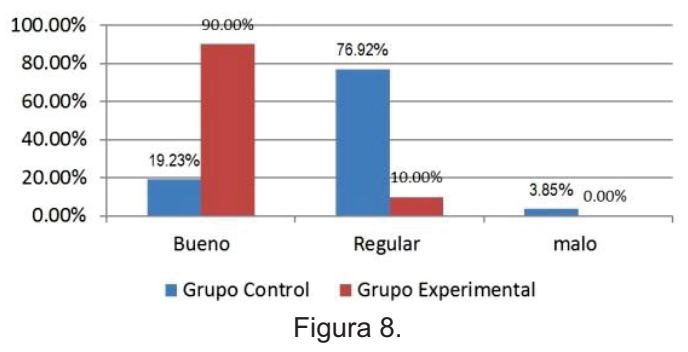

Evaluación de salida de la forma del trabajo de investigación monográfica del grupo control y grupo experimental, Tacna -2012.

De acuerdo a la Figura 8, relacionada a la evaluación de salida de la forma del trabajo de investigación monográfica presentado por los estudiantes del grupo control y grupo experimental; se precisa que el $19.23 \%$ del grupo control y el $90,00 \%$ del grupo experimental se ubican en la categoría bueno; mientras que el $76,92 \%$ y el $10,00 \%$ respectivamente se sitúan en la categoría regular.

La información presentada muestra la presencia de ciertas limitaciones del grupo control en relación a la forma.

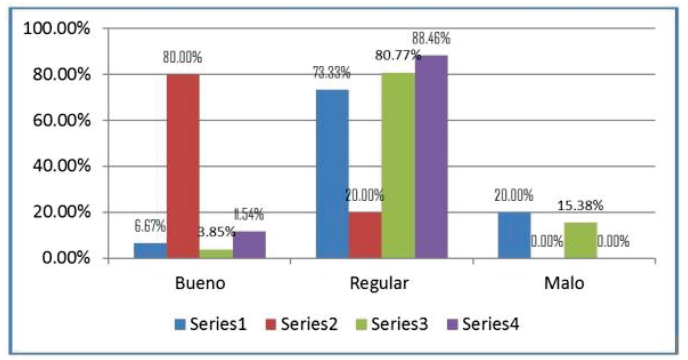

Figura 9.

Consolidado de la relación entre el pre test y post test

De acuerdo a la Figura 9, relacionada a la evaluación de salida y evaluación de entrada del grupo de control y grupo experimental de la calidad del trabajo de investigación monográfica presentado por los estudiantes; se precisa que el $80.00 \%$ del grupo experimental y $11.54 \%$ del grupo de control se ubican en la categoría bueno; mientras que el $20,00 \%$ y el $88,46 \%$ respectivamente, se sitúan en la categoría regular.

La información presentada muestra que ha mejorado significativamente el grupo experimental y una ligera modificación el grupo de control después de la experiencia.

\section{Discusión}

El uso de internet como herramienta educativa y de investigación científica ha crecido aceleradamente debido a la ventaja que presenta el poder acceder a grandes bases de datos y la capacidad de compartir información. Por tanto, es una herramienta fundamental para llevar a cabo una investigación académica.

La investigación planteó la siguiente interrogante ¿En qué medida la aplicación del manual auto instructivo "TCl" permitirá mejorar la calidad de los trabajos de investigación monográfica de los estudiantes de segundo año de LELI de la Escuela Académica Profesional de Educación de la UNJBG de Tacna en el año académico 2012? En el resultado se concluye que efectivamente la calidad de los trabajos de investigación monográfica del grupo experimental presenta una mejoría significativa en relación al grupo de control. Por tanto, en función de los objetivos e hipótesis planteadas, queda verificada y aceptada la hipótesis general. 
El nivel de calidad de los trabajos de investigación monográfica de la mayoría de los estudiantes de segundo año de LELI Y IETI de la escuela de Educación de la UNJBG de Tacna, del grupo control y grupo experimental se ubican en la categoría regular, sin la aplicación del manual auto instructivo "TCl", destacando su mejor desarrollo y acierto en la portada (forma), lo que corrobora la existencia del problema a solucionar.

Sin embargo, con la aplicación del manual auto instructivo "TCl" se ha demostrado la eficacia y eficiencia del manual como estrategia didáctica importante para mejorar la calidad de los trabajos de investigación monográfica de los estudiantes, tal como se puede apreciar en los resultados del grupo experimental (GE) de la presente investigación; con lo cual, se valida dicho manual. Por lo tanto, el manual auto instructivo "TCl" repercute en forma significativa en la calidad de los trabajos de investigación monográfica.

El nivel de calidad de los trabajos de investigación monográfica del grupo experimental de los estudiantes de segundo año (LELI) de la Escuela Académica Profesional de Educación de la UNJBG de Tacna, antes de la aplicación del manual auto instructivo "TCl" son regulares; mientras que después de la aplicación del manual auto instructivo "TCl" mejora significativamente; en cambio el grupo de control presenta solo una leve mejoría en la calidad de los trabajos de investigación monográfica.

La presente investigación ha permitido demostrar cómo se puede mejorar la calidad de los trabajos de investigación monográfica, pues partiendo de un estado inicial deficitario se ha logrado mayor eficiencia en el grupo experimental (GE), gracias a la aplicación del manual auto instructivo "TCl".

Es importante destacar el procedimiento para aplicar el manual auto instructivo "TCl", pues el estudiante debe seguir una serie de etapas para la elaboración del trabajo de investigación monográfica, lo cual permite elaborar una investigación de calidad para un mejor aprendizaje. Los docentes deben proponer nuevas estrategias didácticas para mejorar la calidad educativa en la región Tacna, tomando en cuenta los avances científicos y tecnológicos, tal como lo proponen a nivel institucional la Pontificia Universidad Católica de Chile (2001) y la Universidad de San Pablo (2010).

\section{Referencias Bibliográficas}

1. Wyatt, A. (1995). La magia de INTERNET. México: Ediciones Mc Graw Hill.

2. Vara, A. (2010). ¿Cómo hacer monografías de investigación?, Manual práctico para los estudiantes. Lima, Perú: Ediciones Universidad San Martín de Porres.

3. Pontificia Universidad Católica de Chile (2001). Guía para la Redacción de Citas Bibliográficas. Consultado el 09 de abril de 2012. Disponible en http://biblioteca.utalca.cl/doctos/ pdf/citas_puc.pdf 
4. Universidad de San Pablo (2010). Guía para la elaboración del trabajo monográfico. Consultado el 20 de julio de 2012. Disponible en http://www.sociologiayextension. ecaths.com/archivos/sociologiay extension/guiamonografia.pdf

E-mail: azul10483@hotmail.com

Recibido: $10 / 04 / 14$

Aceptado para publicación:

28/05/2014 